\title{
Quality of Maternity Care at Health Facilities in Eritrea in 2008
}

\author{
Mismay Ghebrehiwet'; Mona Sharan²; Khama Rogo 3; Ogbaselassie Gebreamlak; Berhana Haile ; \\ Mengiestab Gaim6; Zeccarias Andemariam; Shashu Gebreselasie ${ }^{8}$. \\ Institutional Affiliation: \\ ${ }^{1}$ Advisor to the Minster of Health and the Principal Investigator of the study; ${ }^{2}$ World Bank; ${ }^{3}$ World Bank; \\ ${ }^{4}$ Family and Reproductive Health Unit- $\mathrm{MoH}$; ${ }^{5}$ Family and Reproductive Health Unit- $\mathrm{MoH}$; \\ ${ }^{6}$ Eritrean Nurses Association; ${ }^{7}$ Eritrean Nurses Association; ${ }^{8}$ Eritrean Nurses Association.
}

Corresponding Author:

Mismay Ghebrehiwet MD, MPH, PhD. E-mail gmismay@yahoo.com.

Abstract

Objective: To examine the quality of maternal health services at health facilities in Eritrea.

Methods: The study was a cross-sectional survey of all hospitals and health centers and a random sample of a third of health stations. Extensive interviews with health providers and facility managers were undertaken using structured questionaires.

Findings: The key findings of the study include: All hospitals and all health centers provided Basic Obstetric Emergency Care. However, only 11 of the 18 hospitals provided Comprehensive Obstetric Emergency Care including caesarian section. The national referral hospital treated 54 percent of obstetric complications, while health centers and health stations are not proportionally sharing the burden of work.

Recommendations: Eritrean health system which was performing well with the current demand for services can improve its outputs. Upgrading of the function of existing facilities by strengthening the human resource capacity is needed to increase availability of emergency obstetric care by more than one third, using the existing physical structure of health facilities.

Keywords: Maternity care, Quality, availability, accessibility, continuity, management, infrastrScope, Editorial

\section{Introduction}

The concept of quality of health care of patients is as elusive as standards of its measurement. The spectrum of quality has of necessity to be a delicate balance of expectations from the patients, their relatives on one hand, the health service providers and the health institutional expectations (Van den Broek NR, et al, 2009). One of the definitions of quality of care is "the functional effect of an illness and its consequent therapy upon a patient, as perceived by the patient where the domains of quality of life include physical, mental, social, and occupational function; health perceptions; and symptoms of disease" (Swenson JR, et al, 2000).

Quality care should thus lie at the core of all strategies for accelerating progress towards MDG4 and 5. Interventions to measure and improve quality need themselves to be evidence-based as there is variation in the available technological advancements available to mitigate negative outcomes (Faisel H, et al, 2009).

The increasing availability of medical evidence in clinical practice was expected to improve the quality of care. However, this has not been realized. A possible explanation is that quality of care is a complex concept and needs a wider scope starting from the Donabedian triangle of structure, process and outcome to a framework for the analysis of quality of care (Van Driel ML, et al, 2005 ; Wyszewlanski L, et al, 1981).

Access to quality maternity care, including: antenatal care; delivery by skilled attendant, including emergency obstetric care; postpartum and family planning, are key factors in the reduction of maternal and perinatal deaths. Quality of care is defined as the "degree to which health services to individuals and populations increase the likelihood of desired health outcomes and are consistent with current professional knowledge" and it ensures that services are safe, effective, patient-centered, timely, efficient and equitable (Donabedian A, 2003).

The study was undertaken by the Eritrean Nurses Association (ERINA) in collaboration with the Ministry of Health and the World Bank as part of a World Bank intercountry study that was undertaken in three countries, Eritrea, Malawi and Niger in 2008 and 2009.

We attempt, in this study, to examine the capacity of a health system from a quality perspective. A growing body of evidence suggests that quality may be an important determinant of health outcomes. In this study, we gauge quality by expanding the Donabedian quality of health services framework that incorporates structure, process and outcome as elements of quality (Donabedian A, 2003).

- We seek to define quality of maternal health services as a system that encompasses six key dimensions. Elements of structure, process and outcome have been reformulated into dimensions that are relevant to quality of maternal health services, in particular to obstetric care.

As revealed in the conceptual framework depicted in the following figure, the six dimensions include Availability of care, infrastructure, continuity of care, access to a referral system, management and process of care, which collectively define quality of Maternity Care, and has an impact on maternal and perinatal health outcomes. Quality of Maternity Care is known to have an impact on utilization of service. Extraneous factors such as patient condition at admission and care seeking behavior patterns are also known to 


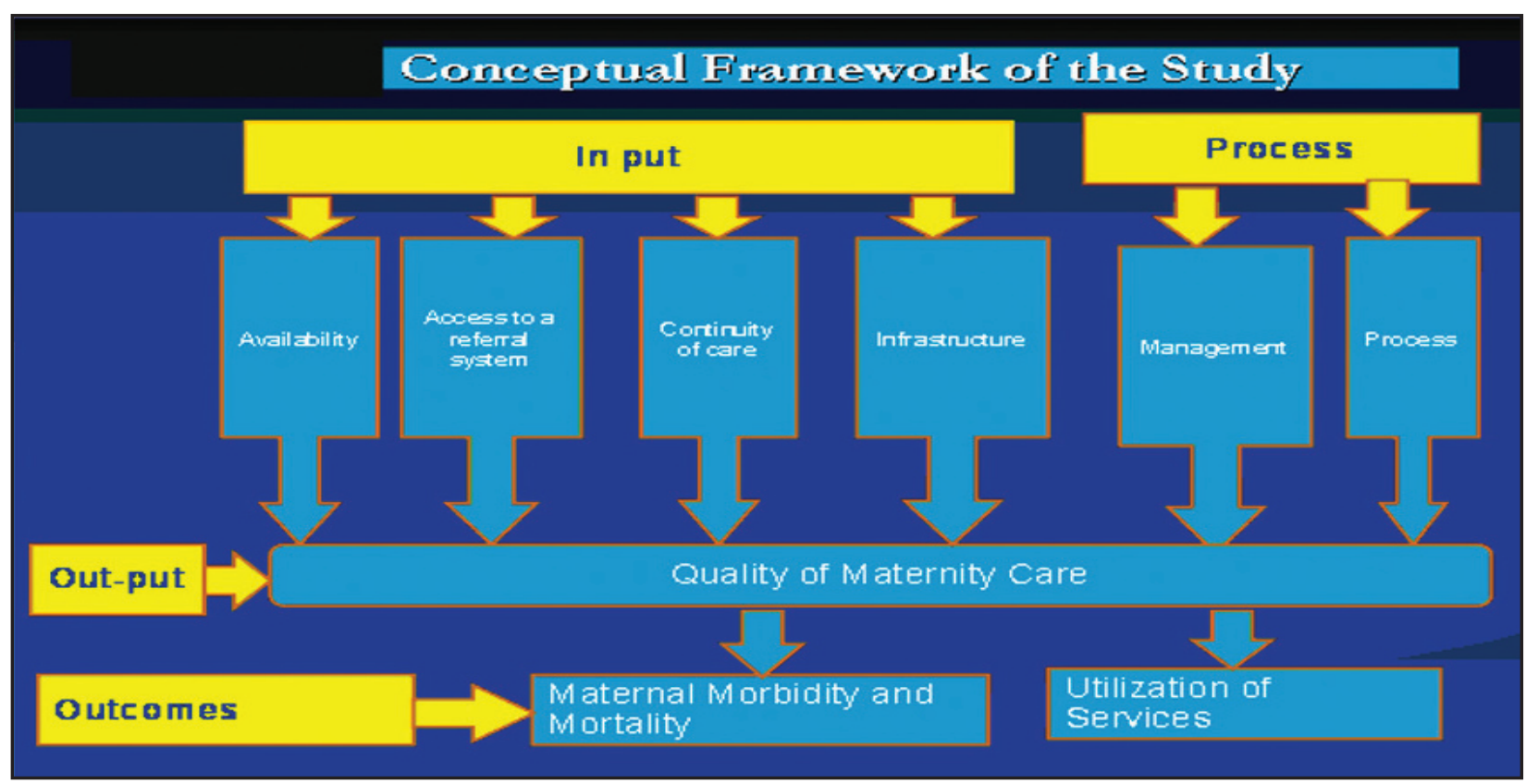

contribute to health outcomes independent of quality of care at the facility, but are not measured in this study.

\section{Materials and methods}

The study was a cross-sectional survey of a nationally representative sample of health facilities. The specific objectives of the study were to determine the quality of obstetric services by examining the structural and process gaps and strengths in the quality of obstetric services and identifying gaps and strengths in quality of care and care utilization.

The survey team collected data from interviews with health providers in each facility. The data were collected from May to July 2008. The spatial distribution of health facilities was determined using GIS to display the location of all health facilities in the sample. The survey team used GPS receivers to record the longitude and the latitude of each health facility that was surveyed. The GIS location of the health facilities was juxtaposed on the map of Eritrea to sow the distribution and location of health facilities.

The sample included all higher level facilities that provided maternity services, including the national maternity referral hospital, all of the six zonal referral hospitals, all sub zonal (community hospitals), all health centers and a random sample of around a third of health stations. A total of 118 facilities (18 hospitals, 47 health centers and 53 health stations) from the six Zobas were included in the sample.

\section{Results}

Availability of care: the spatial distribution of health facilities based on GIS data displayed the location of all health facilities in the sample. The location of the health facilities juxtaposed on the map of Eritrea showing population density by Zoba showed thatmorefacilitiesare concentrated in the densely populated areas. The central zone (Zoba Maekel) was the most densely populated and had the highest concentration of hospitals. The Southern Red Sea Zoba was the most sparsely populated and had the fewest number of health facilities.
At all levels, normal deliveries are mostly conducted by nurse-midwives, nurses and associate nurses. Caesarians are always conducted by obstetricians/ gynecologists, physicians or surgeons. Nurses or nursemidwives do not conduct Caesarian deliveries. Most Health Centers/stations only manage normal deliveries. Health Centers/stations focus on management of normal deliveries, provision of basic EmOC and referral of mothers and newborns with complications. Table 1 summarizes the maternity services that are provided by type of health facility and Zoba, as reported by the health personnel in the facilities.

\begin{tabular}{|l|c|c|c|}
\hline \multicolumn{3}{|l|}{$\begin{array}{l}\text { Table 1: Availability of EmOC functions by type of } \\
\text { health facilities }\end{array}$} & \multicolumn{3}{|l|}{$\begin{array}{l}\text { Percentage distribution of } \\
\text { health facilities providing } \\
\text { the signal function }\end{array}$} \\
\cline { 2 - 4 } & $\begin{array}{l}\text { Hospital } \\
\text { EmoC functions }\end{array}$ & $\begin{array}{l}\text { Health } \\
\text { center } \\
\mathrm{n}=47\end{array}$ & $\begin{array}{l}\text { Health } \\
\text { station } \\
\mathrm{n}=53\end{array}$ \\
\hline Parentral antibiotics & 100.0 & 97.8 & 84.6 \\
\hline Parenteral oxytocin & 100.0 & 93.5 & 67.3 \\
\hline $\begin{array}{l}\text { Other Parenteral sedatives/ } \\
\text { anticonvulsants }\end{array}$ & 100.0 & 89.1 & 50.0 \\
\hline $\begin{array}{l}\text { Manual removal of retained } \\
\text { placenta }\end{array}$ & 100.0 & 89.1 & 50.0 \\
\hline $\begin{array}{l}\text { Removal of retained } \\
\text { products }\end{array}$ & 94.4 & 80.4 & 46.2 \\
\hline Assisted vaginal delivery & 94.4 & 69.6 & 1.9 \\
\hline Breech delivery & 100.0 & 84.8 & 34.6 \\
\hline Blood transfusion & 83.3 & $2.2^{*}$ & NA \\
\hline Caesarian section & 61.1 & NA & NA \\
\hline Magnesium Sulfate & 61.1 & 26.1 & 3.8 \\
\hline $\begin{array}{l}\text { Neonatal resuscitation with } \\
\text { bag and mask }\end{array}$ & 100.0 & 87.0 & 59.6 \\
\hline \multirow{2}{*}{ Digsa health center } & & & \\
\hline & & & \\
\hline
\end{tabular}


All hospitals and all health centers provided basic EmOC functions. Out of the 18 hospitals in Eritrea, Caesariansection was performed at 11 hospitals (61\%) only.

Most essential drugs such as oxytocics, anticonvulsants, antibiotics and pain killers are available at most hospitals. In general, the availability of essential drugs was lower at health centers as compared to the hospitals. Medical supplies such are syringes, needles, scissors, blades, suture materials, gloves, and washing soap were available at all health facilities. The majority of hospitals had equipment needed for maternity care provision, including gynecological exam table, fetoscope, autoclave, speculum, forceps, etc. One-third of hospitals did not have an operating table and anesthesia equipment. As compared to the hospitals, health centers and health stations were not well-equipped. Although, the health centers had basic equipment, they did not have the equipment required to perform complicated procedures, such as caesarian section.

\section{Infrastructure:}

Some of the variables used to assess health facility infrastructure for maternity care provision included the number of beds at the facility, occupancy status of beds in the labor wards, availability of functioning washroom for maternity patients and availability of a functioning laboratory.

The total number of beds at referral hospitals ranged from 80 to 200. The number of hospital beds at community hospitals, health centers and health stations varied greatly by Zoba. Zoba Gash Barka had the largest number of beds at health facilities and Zoba Debubawi Keih Bahri had the fewest. On an average, Zonal referral hospitals had 143 beds, community hospitals had 62 beds and health centers had 23 beds and health stations had 4 beds. Data on the occupancy status of labor wards show that in 83 percent of referral hospitals, all beds are generally occupied and 17 percent had some empty beds. The reverse was true at the community hospitals, 16 percent reported having all beds occupied, while the remaining two-thirds had some empty beds in the labor wards. Health centers are not proportionally sharing the burden of work (all beds occupied in only $4 \%$ of the health centers while many or some beds were usually empty in $87 \%$ (with $40 \%$ many and $47 \%$ some empty beds) of the health centers. Health stations do not normally treat inpatients, except for proposes of delivery or emergency.

All referral hospitals and community hospitals and 85 percent of health centers reported having a functioning laboratory that performed all tests. Fortyone percent of health stations had a laboratory that performed rapid tests only whereas 58 percent of health stations did not have a laboratory. Only 7 of the 118 facilities reported having a functioning blood bank on site. Operation theaters were available at 15 facilities. An intensive care unit for maternity patients and neonates was only available at the National Referral Hospital.

All referral hospitals but only one-third of community hospitals and two-thirds of health centers/ stations had a clean and functioning washroom for maternity patients. Running water is available in 77.1 percent of the health facilities. The majority (86\%) of the health facilities might be considered as having a safe source of water, as half $(50.6 \%)$ of the health facilities have either a piped source of water (35.3\%) or a pump (15.3\%) or both, $18.2 \%$ have protected well and $15.9 \%$ have a tanker. While the remaining $14 \%$ of the health facilities use unsafe source of water, such as unprotected well or open source (most of these health facilities which use unprotected well or open source are health stations). There is need to improve access to functioning washroom in all health facilities except the referral hospitals, particularly in those which admit patients, namely community hospitals and health centers.

Forty six (39.0\%) of the 118 health facilities visited have EELPA electric power supply for 21.35 hours on average. Among those that get EELPA electric power supply, 85 percent get it for 24 hours, while the availability of EELPA electricity for the remaining 15 percent varies from 3 hours (4.3\%), for 6 hours $(8.7 \%)$ to 16 hours $(2.2 \%)$.

Sixty three (53.4\%) of the 118 health facilities visited have a functioning solar power supply. Thirty four $(28.8 \%)$ have a functioning generator for power supply. The majority of the health facilities (86.4\%) have a functioning refrigerator.

\section{Continuity of care:}

We have already noticed that many health facilities do not provide the services that they should provide. However, when they provide, most of them seem to provide the services at night and on weekends as well. The operational time for outpatient services were optimal. Almost all health facilities were open by 8.00 a.m. and the majority stayed open until 6.00 p.m. On an average, non-emergency outpatient care was available at all facilities for 8 or more hours a day. Hospitals provided outpatient care for more than 8 hours a day; while most health center's out patient services were open for less than 8 hours. In-patient care was available at all hospitals and health centers round-the-clock.

Access to a referral system is measured by factors such as referral patterns, by distance to a referral facility from a referring source and the availability and cost of emergency transportation. Referral patterns by facility type indicate that all zoba hospitals and half of the community hospitals refer patients to the national referral hospital. The majority of health centers and health stations refer patients to the zoba hospitals or the community hospitals. The admission patterns suggest that many patients self-refer directly to the hospitals instead of going to health centers, which could cost them precious time and additional money.

Means and availability of emergency transportation and communication at health facilities was measured as one of the indicators for access to a referral system. Once a decision is made to refer a patient from a health facility, the means and availability of transportation and communication become important in reaching 
the referral facility on time.

Only a quarter of the health facilities have a working land line telephone while only one fifth can access 2way radio communication when needed. Availability of telephone or radio transmitter and an ambulance or a vehicle is worst for health stations and health centers, which are the facilities that should refer most and consult and/or inform the referral facility that they are sending a case of obstetric emergency.

\section{The management:}

This dimension captured data on ownership of the facility, the round-the-clock staff coverage at facilities, etc... In addition, data on indicators on staff perceptions, training, motivation and supervision were also collected. The great majority (87.3\%) of health facilities surveyed are owned by the Government, while 12 percent are owned by NGO/church and one hospital is a Parastatal hospital. The Parastatal hospital (Sembel hospital in Asmara) is actually owned by the Ministry of Health but is run as a separate hospital with marketing principles of private for profit. It makes profit and pays taxation. There is no other private hospital or health center in the country but there were around 60 private clinics which were not included in the study.

Staff coverage for day and night shifts was optimal. Health providers were available at the facility on both day and night shifts. Two-thirds of referral hospitals had an obstetrician or gynecologist available round the clock. One-third had a surgeon and a physician available on both the day and night shifts. Only onequarter of community hospitals had an obstetrician and half had a physician available 24 hours a day. No noteworthy discrepancies were observed in the availability of health providers between the day and night shift.

Staff perceptions regarding maternity services at the facility revealed that the large majority of referral hospitals had experienced problems in handling the patient load for maternity care. One-third of referral hospitals always had problems in handling the patient load and 46 percent had problems sometimes. The majority of community hospitals also had problems in handling patient load, but a smaller proportion always had problems as compared to referral hospitals. About 54 percent reported having problems sometimes. Most health centers did not have problems in handling patient load, 32 percent reported having problems sometimes. In contrast almost all (91 percent) of health stations did not have problems in handling patient load, only 6 percent reported having problems sometimes.

According to the respondents, the overwhelming majority of health facilities were inadequately staffed for maternity services. Above 91 percent of referral facilities, 79 percent of community hospitals, 64 percent of health centers and 63 percent of health stations did not have adequate numbers of staff for maternity care provision as perceived by the providers working in maternity care. The perceptions regarding staffing corresponds with perceptions about patient load, indicating a similar pattern of high burden at the referral hospital level, followed by a lower burden at community hospitals and a relatively light load observed at the health centers and health stations.

Asimilargradientwasobserved on questions related to staff workload and job satisfaction. Staff working at the referral hospitals were the most frequently overworked as compared to those working at the lower level facilities. Ninety-two percent of staff working in the maternity ward at the referral hospitals was either sometimes or always overworked. A comparatively lower proportion of staff at the community hospitals (62 percent) ever felt overworked. Health center and health station staff were the least overworked; about a third reported of health center and 46 percent of health station staff having an optimal workload. Fourteen percent of health center staff feel that they did not have much work.

Reported job satisfaction data indicate that staff at the community hospitals were the most satisfied as compared to those at the higher and lower level facilities. About 96 percent of staff at community hospitals was satisfied or highly satisfied with their jobs. A smaller proportion 79 percent in referral hospitals, 91 percent in health centers, 86 percent in health stations reported being satisfied or highly satisfied. The trend reveals that staff dissatisfaction tends to be high when the workload is either too heavy or too light. On the other hand, at the community hospital, where the workload is neither heavy, nor too light, staff appeared to be most satisfied. Staff dissatisfaction with salary was nearly universal. At the referral hospitals, 100 percent of respondents felt they were not satisfied with their salary. At the community hospital level 94 percent reported not being satisfied. A relatively smaller proportion 84 percent at the health center and 81 percent at the health station level, reported not being satisfied with the salary they made.

Staff training and supervision was assessed to determine the quality of human resources for maternity care provision. As a measure of skills maintenance of maternity/midwifery staff, the maternity staff were asked whether they were provided with training in midwifery skills and if so when was the last time the training provided.

Out of 217 respondents, 95 percent had received refresher training on the current job but almost all (99 percent) felt that they needed additional training to perform job responsibilities. About 69 percent of respondents had been trained in life-saving skills (LSS). The national and zonal referral hospitals had the highest number of LSS trained workers, followed by the community hospitals and health centers and stations. On an average, there were 30 trained LSS workers at the referral hospitals, approximately 12 at the community hospitals and 3 at the health centers and stations. Zoba Maekel had the highest number of trained LSS workers, followed by zoba Debub. Zoba Gash Barka had the fewest. Other types of training received by respondents included training on HIV/ AIDS prevention, prevention of mother-to-child transmission of HIV, infection prevention and family planning. 
The large proportion of maternity staff reported being supervised sometimes, followed by regularly at all types of facilities. About 21 percent of staff at the referral hospitals, 27 percent at the community hospitals, 9 percent at the health center and 14 percent at the health station level never received supervision.

\section{The process of care:}

Maternity care provision was measured by variables including, compliance with standards of care and patient discharge and follow-up patterns. There was a high degree of compliance with infection prevention practices at all levels of health facilities. Over 95 percent of staff always used disposable gloves at all levels. The use of disinfectant, soap and decontamination was above 90 percent at all types of facilities. Use of protective wear was lower at health centers as compared to hospitals.

At the referral and community hospitals, maternity patients were visited at least once per day by either doctors or nurses/midwives. Rounds were made at least once a day by nurses/midwives at half of the health centers/stations.

In a majority of the facilities of most zones, results of lab tests were always received on time. Most referral hospitals (83 percent), community hospitals (92 percent), health centers (87 percent), reported that they always received lab test results in a timely manner. As expected, only 43 percent of health stations always received lab results on time, while about 22 percent of health stations do not receive any lab results, as they do not perform lab test.

Patients were usually discharged on the same day after a normal delivery at the referral hospitals. In 42 percent of the community hospitals and 22 percent of the health centers mothers were discharged on the same day after normal delivery. About 57 percent of the health centers and 55 percent of health stations allowed women to stay for a day (overnight) after normal delivery and a small proportion let them stay for two days or more. After Caesarian deliveries, 83.3 percent were discharged from referral hospitals within 4-7 days, while 100 percent were discharged from community hospitals within 5-7 days. Hence, the length of stay was relatively short at the referral hospitals as compared with the community hospitals. No Caesarian deliveries are performed in health centers or health stations. While the referral hospitals discharged Caesarian-section patients after 4-5 days, the community hospitals allowed them to stay for 5-7 days.

\section{Discussion:}

The study findings cover a detailed and compre hensive picture of the quality of Maternity Care in Eritrea. The findings reflect both the challenges and the achievements of the Eritrean health system and the implications they have for improving maternal health and survival in the country.

The spatial variation in the locations of health facilities indicated that hospitals are not evenly distributed by geographical location. Hence, functionally and structurally upgrading health centers to serve as community hospitals can drastically improve the availability of comprehensive emergency obstetric and other hospitals services.

Based on the WHO, UNICEF and UNFPA 1997 classification, the national referral hospital and all of the five Zonal referral hospitals studied perform all of the nine functions and could be classified as comprehensive EmOC facilities. As the national referral hospital and the Sembel Parastatal hospital are located in Zoba Maakel (the capital, Asmara), this means all zones have at least one comprehensive EmOC facility.

According to the assessments done in 2003 and 2007, Eritrea has the UN recommended number of EmOC facilities and almost the recommended number of BEmOC facilities (Ghebrehiwet and Morrow 2006, Laverentz 2007). Understandability there is inherent limitation in applying global standards, as they don't take into account country specific conditions. However, despite the limitation, the UNICEF, WHO and UNFPA standard as a rough guide, this study also shows that the country has been able to meet the required adequate number of $\mathrm{EmOC}$ facilities as per population size but the geographical distribution is not yet equitable. It is encouraging to note that most health facilities in Eritrea have at least the minimum acceptable level the essential equipments, supplies and drugs for the functions they perform.

Bed occupancy rate is high at the level of referral hospitals; medium at level of community hospitals; and low at $\mathrm{HC} / \mathrm{HS}$ level. This is an indication of underutilization of the service and existence of an opportunity for the improvements in institutional (health facility) delivery by skilled attendants and filling the gaps in human resource; power supply; availability of clean and functioning washrooms etc.

The strategy for preventing maternal mortality rests on the system of referrals. It is expected that women will go to the nearest health facility for delivery. However, most patients actually directly go to hospitals for obstetric complications. Patients appear to be making rational decisions in choosing to go to the referral hospitals where doctors are available instead of going to health centers and health stations, where they are not likely to get treated for serious complications. Providers at health centers and health stations also prefer to refer patients with serious complications to hospitals than risk treating them on site given the minimal support and clinical expertise that is available at that level.

Once a decision is made to refer a patient from a health facility, availability and cost of transportation and distance to the referral center become important in reaching the referral facility on time. This study revealed that although transport is available, distances and travel times are long. Hence, for patients in critical emergencies, the referral linkages are less than optimal. In addition to availability and cost of transportation, availability of a communication system becomes important, both in looking for options of transportation and for informing referral facilities so that they get prepared in anticipation for arrival of a referred emergency patient. 
Availability of telephone or radio transmitter and an ambulance or a vehicle is worst for health stations and health centers, which are the facilities that refer most and should consult and/or inform the referral facility that they are sending a case of obstetric emergency. Hence, there is need of equipping these facilities with a means of communication to higher facilities, such as mobile or land line telephone or radio communication.

Due to the large volume of patients coming to the referral facilities, the staffs in the referral hospitals are under tremendous pressure. Providers at the health centers and health stations, on the other hand, do not get a large number of patients so are not proportionally sharing the burden of delivering mothers.

The national safe motherhood protocol states the importance of regular and frequent supervision (at least every 3 months) at lower operational levels (health stations and health Centers) and somewhat less frequent supervision (at least twice per year) at higher (Community and Zonal hospital) levels. The maternity care is far from meeting these supervisory targets.

The findings clearly reveal the pressure on the infrastructure and the service providers. Although the referral hospitals are working well, they are stretched to capacity. Patient discharge patterns indicate that the referral hospitals are trying to accommodate the huge demand by discharging patients as soon as possible. Since a higher proportion of obstetric complications develop in the postpartum period, a short hospital stay post-delivery may not be optimal.

The Maternity Care in Eritrea has strengths which make it unique and exemplary in many ways. The process of care provision is excellent. There is a high degree of compliance with infection prevention and other standards of care. Drugs, supplies and equipment are usually adequate. Staff coverage is available round-the-clock, patient follow-up in the wards and postpartum care is also notably high.

Our findings show that the Eritrean Maternity Care is overloaded at the National and Zonal Referral Hospitals, but is performing well with the current demand for services. The low bed occupancy rate at the level of community hospitals, HCs and HSs indicate that the system could absorb more clients provided the human resource constraint is addressed in a more effective manner. The study findings have also highlighted several gaps in the health system that have a direct bearing on the quality of maternity care in the country. The shortage of clinical service providers is the biggest constraint facing the maternity care at the moment. However, with the recent and upcoming graduation of medical doctors and other health professionals, this is expected to be filled soon.

\section{Conclusion and Recommendation}

Over all it can be concluded that the Eritrean Maternity Care is performing well with the current demand for services. The key recommendations of the study include: - upgrade function of existing facilities by filling the human resource and other gaps. By doing so, the availability of emergency obstetric care could be increased by more than one third, using the existing physical structure of health facilities. Other recommendations include: - filling the shortage of human resource at every level; establish and strengthen supervisory support services at all levels; sustain and improve further the existing good quality of maternity care provision and management.

\section{Acknowledgement}

The Eritrean Nurses Association would like to express its gratitude to the Ministry of Health and the World Bank for choosing and trusting ERINA to do the important job and for all the valuable assistance they rendered. It would also like to express its gratefulness to the Africa Human Development of the World Bank and the Bank-Netherlands Partnership program for sponsoring the important intercountry study. It would like to express its gratitude to the author who is the principal investigator of the study and to the co-authors for the excellent job. It is indebted to all Executive Committee Members of ERINA and other ERINA members who enthusiastically worked as Research Coordinators, Research Supervisors, Data Collectors, and Managers as well as in many other functions of the study.

\section{Reference}

1. Donabedian, A. An introduction to quality assurance in health care. New York, Oxford University Press; 2003.

2. Faisel H, Pittrof R, El-Hosini M, Habib M, Azzam EUsing standard primipara method to compare the quality of maternity care in Cairo and London. J Obstet Gynaecol. 2009;29(4):284-7.

3. Ghebrehiwet Mismay, Richard H. Morro, Availability, Utilization and Quality of Normal Delivery and Emergency Obstetric Care in Eritrea. Journal of Eritrean Medical Association (JEMA), Vol II. No. 1, Dec. 2007, Asmara, Eritrea.

4. Gottlieb, P. and G. Lindmark (). "WHO indicators for evaluation of maternal health services, applicability in least developed countries: a case study from Eritrea." African Journal of Reproductive Health 2002; 6(2): 13-22.

5. Institute of Medicine Crossing the quality chasm: A new health system for the 21st century. Washington DC, National Academy Press; 2001.

6. Ministry of Health Eritrea, Annual Health Service Reports, HMIS and R\&HRD, MOH; 2000.

7. Ministry of Health Eritrea, Annual Health Service Reports, HMIS and R\&HRD, MOH; 2008.

8. Swenson JR, Clinch JJ. Assessment of quality of life in patients with cardiac disease: the role of psychosomatic medicine. J Psychosom Res. 2000;48(4-5):405-15.

9. Van den Broek NR, Graham WJ. Quality of care for maternal and newborn health: the neglected agenda. BJOG. 2009;116 Suppl 1:18-21.

10. Van Driel ML, De Sutter Al, Christiaens TC, De Maeseneer JM. Quality of care: the need for medical, contextual and policy evidence in primary care. J Eval Clin Pract. 2005;11(5):41729.

11. WHO, UNICEF, and the World Bank. Maternal Mortality estimates for 2005. New York 2007.

12. Wyszewianski L, Donabedian A.Equity in the distribution of quality of care. Med Care. 1981;19(12 Suppl):28-56. 